\title{
Cardiac metastasis from liposarcoma to the right ventricle complicated by massive pulmonary tumor embolism
}

\author{
Metástasis cardíacas por liposarcoma a ventrículo derecho complicado con embolismo \\ tumoral pulmonar masivo
}

\author{
Mateo Porres-Aguilar ${ }^{1 *}$, Ignacio De Cicco², Javier E. Anaya-Ayala ${ }^{3}$, Mateo Porres-Muñoz ${ }^{4}$, \\ Luis E. Santos-Martínez ${ }^{5}$, César A. Flores-García ${ }^{6}$ and Héctor Osorio ${ }^{7}$
}

${ }^{1}$ Internal Medicine Clinic and Professional Associates; ${ }^{2}$ Division of Cardiology, University of Texas Health Sciences Center at San Antonio. San Antonio, Texas, USA; ${ }^{3}$ Division of Vascular Sugery and Endovascular Therapy, Instituto Nacional de Ciencias Médicas y Nutrición Salvador Zubirán, Mexico City; ${ }^{4}$ Department of Internal Medicine; Beneficencia Española de Tampico; Tampico; ${ }^{5}$ Departamento de Hipertensión Pulmonar y Corazón Derecho, Hospital de Cardiología, Centro Médico Nacional Siglo XXI, Instituto Mexicano del Seguro Social, Mexico City; ${ }^{6}$ Department of Pathology, Centro Médico Nacional Siglo XXI, Instituto Mexicano del Seguro Social, México City. México; ${ }^{7}$ Division of Cardiology; Methodist Healthcare Heart Hospital, San Antonio, Texas, USA.

Liposarcomas represent the second most common type of soft tissue sarcomas after malignant fibrous histiocytoma. There are four histological subtypes according to the World Health Organization: well-differentiated (atypical lipomatous) liposarcoma, dedifferentiated, myxoid (round-cell) liposarcoma (ML), and pleomorphic liposarcoma ${ }^{1}$. Their primary anatomical sites of origin from liposarcomas are the retroperitoneum in $25-50 \%$ and the lower limbs in $25-35 \%$. The most frequent distant sites for metastasis arising from $\mathrm{ML}$ include lungs, retroperitoneum, abdominal cavity, and chest wall'. ML accounts for $30-50 \%$ of all liposarcomas, with a peak incidence between the third and the fifth decade of life, being more frequent in males than females, exhibiting an overall metastatic rate of $30 \%$. Interestingly, it has an expansive growth pattern, with less aggressive infiltrative behavior, thus, causing frequently obstructive clinical syndromes at the main site of metastasis, however, metastatic intracardiac soft tissue sarcomas represent an extremely rare clinical entity ${ }^{1}$. Some authors believe that in patients with a known history of liposarcoma, the time interval between diagnosis and the occurrence of distant metastatic disease ranges from 3 to 25 years ${ }^{1}$.

Fulminant right ventricular (RV) failure from RV metastasis causing outflow obstruction, cardioembolic complications, such as ischemic stroke or significant massive pulmonary tumor embolism (PTE) could occur, carrying catastrophic consequences ${ }^{2-6}$. PTE complicating RV metastasis from ML represents an extremely difficult premortem diagnostic challenge for clinicians. Herein, we describe, to be best of our knowledge, the first case, of a rapidly progressive hematogenous metastatic ML to the RV complicated with extensive PTE with catastrophic outcomes.

\section{Case description}

A 63-year-old Hispanic woman with medical history of hypertension and hyperlipidemia was transferred to 
our hospital, with a significant history of 5-week progressively worse dyspnea; in the previous hospital, she was diagnosed with pneumonia; however, despite antibiotics given, her condition started to deteriorate over the following weeks. The patient also reported unknown quantity of weight loss in the past 4 months, as well as progressively worse right hip pain and discomfort with subjective swelling for the past 2 months, provoking her a couple of falls.

On physical examination, her blood pressure was $105 / 70 \mathrm{mmHg}$, heart rate at 105 beats per minute, afebrile with an oxygen saturation of $94 \%$ at $4 \mathrm{~L} / \mathrm{NC}$; cardiac auscultation revealed significant tachycardia; lung auscultation with bilateral lungs clear; right hip with pain on deep palpation, and exacerbation of pain on extension and flexion of it; and no skin discolorations noted.

Laboratory findings were notable for severe thrombocytopenia of 25,000 cells $/ \mathrm{mm}^{3}$ (normal $>140,000$ cells/ $\mathrm{mm}^{3}$ ) and lactate dehydrogenase of $1042 \mathrm{U} / \mathrm{L}$ (normal $<250 \mathrm{U} / \mathrm{L}$ ). 12-lead electrocardiogram showed sinus tachycardia. Chest radiography showed bilateral basal multifocal airspace opacities, suggesting possible infarcts or atelectasis. Bilateral lower extremity venous Doppler ultrasonography did not reveal any deep vein thrombosis.

Transthoracic echocardiography showed a "D"-shaped morphology of the left ventricle during systole, left ventricular ejection fraction between $60 \%$ and $65 \%$; moderate right atrial enlargement, severe RV dilation, and hypokinesis with positive McConnell's sign, with multiple intraventricular echodensities, suggestive of $\mathrm{RV}$ thrombi, some of them pending in the posterior leaflets of the tricuspid valve, and other attached to the free wall of the RV, biggest thrombi dimensions are $1.5 \mathrm{~cm} \times 1.7 \mathrm{~cm}$; lack of microbubble (transpulmonary) contrast agent uptake, suggesting possible avascular structures, estimated pulmonary arterial pressure at $61 \mathrm{mmHg}$ (Fig. 1A).

Computed tomographic (CT) angiography of the chest for pulmonary embolism protocol showed central right and left main bilateral pulmonary emboli, extending into the lobar arteries; multiple thrombi filling the $\mathrm{RV}$, with bilateral diffuse lower lobes ground glass opacities (Fig. 1B). CT with contrast of the abdomen and pelvis showed extensive peripherally enhancing fluid collections within the right hip and right thigh musculature, measuring $14.3 \mathrm{~cm} \times 14.8 \mathrm{~cm}$. Pathologic macroscopic and microscopic descriptive findings are shown (Fig. 2A-2F).

During her hospital course, she was managed with intravenous argatroban drip, given her severe consumptive

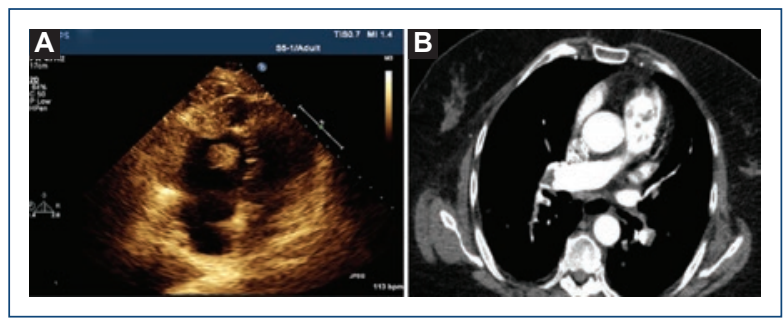

Figura 1. Transthoracic echocardiogram at apical fourchamber view showing severe right ventricular (RV) dilation, presence of the McConnell sign (significant distal RV lateral free wall hypokinesis) suggestive of multiple RV thrombi; biggest thrombi dimensions are $1.5 \mathrm{~cm} \times 1.7 \mathrm{~cm}$ (A). Computed tomographic angiogram of the chest showing intracavitary RV thrombi with right main and left lobar bilateral pulmonary emboli extension, and absence of contrast enhancement within the filling defects (B). Please, also, see supplementary videoclip of the transthoracic echocardiography in the electronic version of this article.

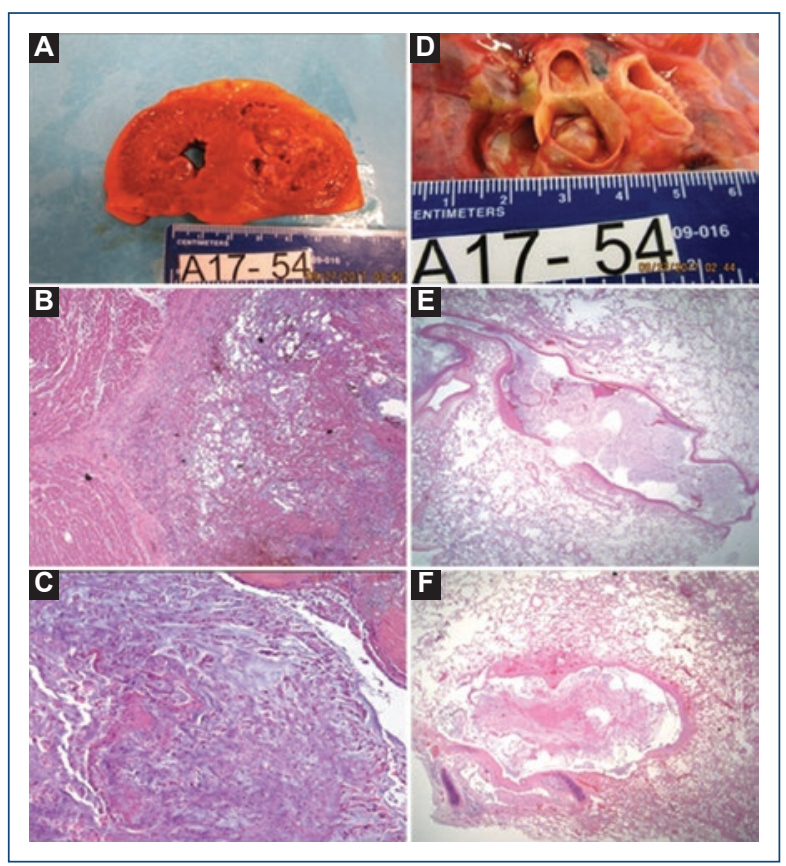

Figura 2. Gross fresh appearance, short axis section. Right ventricle occupied by tumor (A). Low-power field, the tumor invades endocardium and cardiomyocytes H\&E $\times 10$ (B). Conspicuous myxoid stroma and malignant cells with "floating" aspect H\&E $\times 25$ (C). Pulmonary artery filled by tumoral thrombus (d). Histological view tumoral thrombus and lung parenchyma, H\&E $\times 10$ (E). Acute pulmonary thromboembolism concomitant H\&E $\times 10$ (F).

thrombocytopenia. Orthopedic surgery performed a fine-needle aspiration biopsy on her right hip, given her extensive lesions concerning for malignancy, and given her very poor surgical candidacy and unacceptable 
high risk for major bleeding events. The patient's clinical condition rapidly deteriorated in the next 24-48 h, to the point that she became severely hypoxemic, hypotensive, requiring mechanical ventilatory support, and vasopressor therapies. Unfortunately, the patient passed away, after her family members decided to withdraw care through her $4^{\text {th }}$ day of hospital stay.

\section{Discussion}

Although primary cardiac tumors are rare (between $0.01 \%$ and $0.1 \%$ in postmortem studies), the frequency of cardiac metastasis is more common, ranging between $0.5 \%$ and $3.7 \%$ in the general population; ${ }^{2-4}$ however, its frequency in patients with known malignancy varies from $9.1 \%$ to $14.5 \%$ in patients with advanced metastatic malignancies ${ }^{4,5}$. The symptomatology from cardiac metastasis is non-specific, and the clinical features are highly variant and dependent on the location and disseminated tumor burden ${ }^{2-5}$.

Cardiac metastasis can spread to the heart mainly by four mechanisms: direct extension or contiguity, hematogenous spread, lymphatic spread or by transvenous and/or intracavitary invasion ${ }^{4,5}$. Melanomas, lymphomas and sarcomas are amongst the most common tumors that cause hematogenous invasion. Certain malignancies such as renal cell carcinomas and hepatocarcinomas can extend into the inferior vena cava and right atrium, through transvenous diffusion ${ }^{6}$.

Pericardial metastasis is the most common site of invasion, with an estimated frequency of $60-69 \%$ of all necropsy studies; epicardial and myocardial involvement represent the second and third most common sites, in approximately $25-34 \%$ of cases; endocardial and intracavitary are rare, representing $3-5 \%$ on necropsy studies; however, they can present with exaggerated and life-threatening clinical features with immediate negative outcomes ${ }^{2-6}$. Cardiogenic shock due to fulminant RV failure from RV metastasis causing outflow obstruction, significant cardioembolic complications from tumor embolism, causing ischemic stroke or pulmonary tumor emboli, like in our patient, can carry catastrophic outcomes.

Intracardiac metastatic solid sarcomas represent extremely rare clinical entities. From $<40$ cases of metastatic cardiac ML that has been reported in medical literature in English language since 1968, only 13 cases have been reported in the medical literature involving the RV since the first case reported by Godwin et al., in $1981^{7,8}$.
In an imaging-based review series, Chiles et al. ${ }^{9}$ reported an incidence of $0.6 \%$ of intracardiac metastases from sarcomas, with an increased incidence in males, the primary $\mathrm{ML}$ usually located in the lower extremities, with a long interval between the occurrence of the primary ML and metastasis (usually months to years), indicating slow tumor growth, although, in our patient, she had a subacute clinical presentation, with relatively rapid progression and clinical deterioration.

Among many diagnostic imaging techniques currently available for the evaluation of cardiac metastasis, contrast-enhanced transthoracic echocardiography typically represents the best initial diagnostic imaging modality of choice ${ }^{9,10}$.

Macroscopic PTE has been reported in soft tissue sarcomas, hepatocellular, breast, and renal cell carcinomas, whereas microscopic PTE has been found in gastric, hepatocellular, pancreatic, and choriocarcinomas ${ }^{11}$. Tumor embolism possesses a significant level of resistance to recanalization, leading to progressive and irreversible vascular obstruction. Even in patients with a history of known malignancy, accurate diagnosis is only made in as few as $6 \%$ antemortem ${ }^{11}$. PTE has a frequency of $0.3-26 \%$ of solid malignancies in necropsy studies, with a mortality rate of approximately $8 \% .{ }^{11}$ Latchana et al. ${ }^{12}$ performed a focused literature review with a particular focus of soft tissue sarcomas and PTE, finding 45 cases, in which 14 cases arise from soft tissue sarcomas and 31 cases from bone sarcomas; the most common symptom was dyspnea $(87 \%)$, followed by cough $(29 \%)$, chest pain $(7 \%)$, and hemoptysis (4-5\%); most of the patients presented acutely (0-2 weeks), seven patients presented subacutely (2-8 weeks), and only one patient had a chronic presentation (>8 weeks).

\section{Conclusions}

Metastatic hematogenous $\mathrm{ML}$ involving the RV with extensive/massive PTE represents an extremely rare clinical entity, with significant challenges while trying to establish the diagnosis premortem. Even in the early stages of the disease, despite offering prompt and adequate therapies for ML, prognosis has been generally poor, even in the short term. In this particular case, PTE turned out to be subacute in onset with rapid progression, with extremely difficult premortem diagnosis. This case is extremely rare, authentic, and unique because, to the best our knowledge, this is the first case in which its clinical presentation was compatible with extensive/ 
massive PTE, complicating RV metastasis from ML, and ending up with devastating consequences.

\section{Conflicts of interest}

None.

\section{Funding}

None.

\section{Ethical disclosures}

Protection of human and animal subjects. The authors declare that no experiments were performed on humans or animals for this study.

Confidentiality of data. The authors declare that no patient data appear in this article.

Right to privacy and informed consent. The authors declare that no patient data appear in this article.

\section{References}

1. Orvieto E, Furlanetto A, Laurino L, Dei Tos AP. Myxoid and round cell liposarcoma: a spectrum of myxoid adipocytic neoplasia. Semin Diagn Pathol. 2001;18:267-73.

2. Goldberg AD, Blankstein R, Padera RF. Tumors metastatic to the heart Circulation. 2013;128:1790-4.

3. Hudzik B, Miszalski-Jamka K, Glowacki J, et al. Malignant tumors of the heart. Cancer Epidemiol. 2015;39:665-72.

4. Bussani R, De-Giorgio F, Abbate A, Silvestri F. Cardiac metastases. J Clin Pathol. 2007;60:27-34.

5. Bruce CJ. Cardiac tumours: diagnosis and management. Heart. 2011 97:151-60.

6. Santos Martínez LE, Martínez Reding J, Alfaro JK, et al. Severe right ventricular hypertension secondary to metastatic obstruction due to hepatocarcinoma. Arch Cardiol Mex. 2005;75:170-7.

7. Godwin JD, Axel L, Adams JR, et al. Computed tomography: a new method for diagnosing tumor of the heart. Circulation. 1981;63:448-51.

8. Motevalli D, Tavangar SM. Extensive left ventricular, pulmonary artery, and pericardial metastasis from myxoid liposarcoma 16 years after the initial detection of the primary tumour: a case report and review of the literature. Malays J Pathol. 2017;39:201-5.

9. Chiles C, Woodard PK, Gutierrez FR, Link KM. Metastatic involvement of the heart and pericardium: CT and MR imaging. Radiographics. 2001; 21:439-49.

10. Mankad R, Herrmann J. Cardiac tumors: echo assessment. Echo Res Pract. 2016;3:R65-R77.

11. Jorens PG, Van Marck E, Snoeckx A, Parizel PM. Nonthrombotic pulmonary embolism. Eur Respir J. 2009;34:452-74.

12. Latchana N, Daniel VC, Gould RW, Pollock RE. Pulmonary tumor embolism secondary to soft tissue and bone sarcomas: a case report and literature review. World J Surg Oncol. 2017;15:168. 